\title{
Review
}

\section{Review of Research on the Prevention of HPV Infection and Cervical Cancer}

\author{
Tao Wang
}

Department of Pharmacy, Tianjin Central Hospital of Gynecology and Obstetrics, Tianjin China

\author{
Keywords \\ HPV; cervical cancer; infection \\ Correspondence \\ Tao Wang, \\ E-mail: Wangtg|@sina.com
}

DOI: 10.1515/ii-2017-0128

\begin{abstract}
Worldwide, cervical cancer remains as one of the most common malignancies that threaten women's health. An epidemiological survey has shown that high-risk chronic HPV infection is the leading cause of cervical cancer. Thus, the prevention of HPV infection is the main approach to the prevention and treatment of cervical cancer. Sexual behavior, individual immunity, and age are important cofactors in the promotion of HPV infection. Preventive measures that have been gradually implemented worldwide have significantly decreased the incidence of cervical cancer in recent years. These measures include preventive vaccination against common high-risk HPV and cervical cancer screening, which includes HPV testing. HPV testing in cervical cancer screening has received increased attention and provides the basis for the prevention and treatment of cervical cancer. Given that HPV infection rates significantly vary from region to region, procedures for cervical cancer screening should be improved based on regional differences.
\end{abstract}

Cervical cancer is a unique cancer given its clear cause and preventability. Nevertheless, cervical cancer remains a common malignancy that seriously threatens women's health. Worldwide, the incidence of cervical cancer ranks third and is second only to those of colorectal and breast cancers. The mortality rate of cervical cancer ranks fourth among other malignant tumors and is second only to those of colorectal, lung, and breast cancers. In 2008, international cancer-related agencies reported that annually, 529,512 women are diagnosed with cervical cancer and that 274,967 women die of cervical cancer ${ }^{[1]}$. Denny ${ }^{[1]}$ reported a gap between the incidence and mortality of cervical cancer in different regions. The incidence of cervical cancer in low-income countries and regions is nearly 10 times that in developed countries and regions. An epidemiological survey showed that high-risk HPV infection is the leading cause of cervical cancer. Alam et al ${ }^{[2]}$ reported that $99.7 \%$ of tissue samples from cervical cancer cases are positive for HPV infection and that more than $50 \%$ of cervical cancer cases are co-infected with HPV16 or HPV18. Studies on HPV infection have expanded in recent years. Measures for the prevention and control of HPV have been gradually implemented and have helped decrease the incidence of cervical cancer.

\section{HPV characteristics}

\section{Molecular biology characteristics of HPV}

HPV, a pathogen associated with cervical neoplasms, belongs to the Papillomavirus genus of the papilloma multinodular vacuolar virus family. It is a naked, small, double-stranded RNA virus with a simple structure. HPV DNA encodes 58 open reading frames: E6, E7, E1(E8), E2, E4(E3), and E5. These ORFs are involved in the replication, transcription, translation, regulation and cell transformation of viral DNA. HPV is widely distributed in humans and animals, and has a high tissue and host specificity ${ }^{[3]}$.

\section{Type of HPV}

More than 115 HPV genotypes have been identified. Approximately $40 \mathrm{HPV}$ genotypes are associated with genital tract infection ${ }^{[4]}$. HPV is classified as high-risk HPV, possible high-risk HPV, and low-risk HPV based on structure, function, and pathogenicity ${ }^{[5]}$. The degree by which different HPV subtypes increase the risk of cervical cancer is different. 
HPV16, 18, 31, 33, 45, 52 and 58 are closely associated with the incidence of cervical cancer and are considered as highrisk HPV subtypes. HPV16 and HPV18 account for more than $70 \%$ of HPV infection cases. HPV35, 39, 51, 58, 59, 68, 73 , and 82 are related with the incidence of cervical cancer and are possible high-risk HPV subtypes. Low-risk HPV subtypes include HPV6, 11, 40, 42, 43, 44, 54, 61, 70, 72, 81 , and 89 , and mainly cause benign proliferative lesions of skin mucosa, such as genital papilloma, exogenous genital warts and lesions, and low cervical intraepithelial neoplasia (CIN) ${ }^{[6]}$. HPV26, 53 and 66 have received limited research attention.

HPV16 is closely associated with cervical cancer and is present in more than $50 \%$ of cervical cancers. HPV 16 variants have attracted increasing attention in recent years. HPV16 variants include six variants: European, Asian, Asian-American, North American, African-1, and African-2. Sanchez et al ${ }^{[7]}$ confirmed that infection by HPV16 variants account for more than half of all cervical cancers. In addition, various variants exhibit different potentials to cause cervical cancer. For example, the European and Asian subtypes can increase the incidence of cervical squamous cell carcinoma by three times.

\section{Relationship between HPV and cervical cancer and characteristics of HPV infection}

HPV is ubiquitous in the population: $40 \%$ of women will be infected with HPV during the first two years of their sexually active lives, and $80 \%$ of women over 50 years of age are infected with $\mathrm{HPV}^{[8]}$. However, $70 \%$ and $90 \%$ of the HPV load is automatically cleared by the body's immune system during the first year and the first two years after infection, respectively. Three years after infection, almost only $5 \%$ of the viral load is characterized as continuous infection, and then develops into cervical cancer ${ }^{[9]}$. Denny ${ }^{[1]}$ believes that under the influence of persistent HPV infection, precancerous cervical lesions require 10-30 years to develop into cervical cancer.

According to an international study conducted by the International Cancer Research Association, HPV16 (53\%), HPV 18 (15\%), HPV45 (9\%), HPV31 (6\%), and HPV33 (3\%) are the most common HPV subtypes in the world. HPV16 is the subtype with the highest infection rate among all cervical lesions ${ }^{[10]}$. Denny ${ }^{[1]}$ reported that the HPV16 subtype is more likely to develop into cervical squamous cell carcinoma, and that the HPV18 subtype is more likely to develop into cervical adenocarcinoma and adenosquamous carcinoma. Harper ${ }^{[9]}$ reported that HPV16 (62\%), HPV18 (8\%), HPV45 (6\%), HPV31 (5\%), and HPV33 (4\%) are the most common HPV subtypes of cervical squamous cell carcinoma. Nearly $90 \%$ of cervical adenocarcinoma cases are positive for infection with the HPV16, HPV18, and HPV45 subtypes.

According to the study data obtained by Vinodhini et al ${ }^{[11]}$ in 2012, the worldwide HPV infection rate among females is $32.1 \%$. The HPV infection rate in developing countries is $42.2 \%$, which is $22.6 \%$ higher than that in developed countries. In Asia, especially eastern Asia (mainly including China and South Korea), the HPV infection rate is $57.7 \%$. Europe exhibits the lowest HPV infection rate: western Europe exhibits the lowest infection rate at $3.7 \%$, followed by southern Europe at $15.1 \%$. In more developed countries, the most common HPV subtypes are HPV16, 18, 58, 52, and 31.

\section{Risk factors for HPV infection}

\section{Sexual behavior}

Sexual behavior is the most important cofactor in the promotion of HPV infection and cancer incidence. Sexual behavior provides patients with direct exposure to HPV. High-risk sexual behavior includes unprotected sex, multiple sexual partners, and young age during sex for the first time ${ }^{[11]}$. High-risk sexual behavior can maintain high viral loads in patients. In addition, the chemical composition of semen directly stimulates the cervix. Thus, up to $60 \%$ of sexually active women are infected with HPV ${ }^{[12]}$.

\section{Individual immunity}

Low individual immunity is another crucial factor in the promotion of HPV infection. Immunodeficient individuals cannot clear HPV infection in a timely manner and are at increased risk for persistent HPV infection. Given that HIVpositive individuals have significantly lower T-cell counts than healthy individuals, the duration of development from cervical precancerous lesions to cervical cancer is significantly shorter, and incidence and mortality are higher ${ }^{[13]}$. HIV-positive individuals are more susceptible to infection by uncommon HPV subtypes. Rahman et al. ${ }^{[14]}$ investigated the incidence of HPV among HIV-positive individuals and found that most common HPV subtypes are HPV52 (10.5\%), 
HPV39 (9.5\%), HPV54 (9\%), HPV45 (7.8\%), HPV56 (7.2\%), HPV53 (6.4\%), HPV31 (6.2\%),HPV42 (5.3\%),HPV16 (5.1\%), HPV51 (5.1\%), and HPV68 (5.1\%).

\section{Age}

Age is another important factor for the promotion of HPV infection. Women of reproductive age are more likely to be infected with $\mathrm{HPV}{ }^{[11]}$. Gita et al. ${ }^{[12]}$ found that the incidence of HPV infection is highest among women aged 35 to 44 . Cercato et al. ${ }^{[15]}$ utilized questionnaires to identify the risk factors for HPV infection. Education, socioeconomic status, gynecological history, use of oral contraceptives, sexually transmitted diseases, working conditions, low body mass, low intake of vegetables, male factors, smoking, and drinking were included as items in the questionnaires.

\section{Prevention of HPV and cervical cancer}

Since 2009, the incidence of cervical cancer has gradually decreased in Western Europe and the United States. Nadarzynski et al ${ }^{[16]}$ proposed that this decrease mainly benefitted from two effective preventative measures: 1) The preventative vaccination of populations at high risk of HPV, including 12-13 year old girls. 2) The universal implementation of cervical cancer screening and HPV testing among women aged 25 to 64 .

\section{HPV vaccine}

Vaccines specific for several HPV subtypes have been developed since the beginning of the $1990 \mathrm{~s}^{[6]}$. At present, two HPV prophylactic vaccines have been developed, including Cervarix and Gardasil. Cervarix is a HPV16 and 18-type divalent vaccine, whereas Gardasil is a HPV6, 11, 16, and 18-type quadrivalent vaccine. Both vaccines are licensed by the US Food and Drug Administration and the European Medicines Evaluation Agency ${ }^{[17]}$.

The period of sexual development is the most appropriate time for preventative vaccination against HPV. The Advisory Committee on Immunization Practice and Department of Obstetrics and Gynecology, American University, believe that HPV-infected individuals should be actively inoculated with HPV vaccines whether their Pap smears are abnormal or their HPV test results are positive ${ }^{[18]}$.

A clinical randomized controlled trial reported by Harper ${ }^{[9]}$ confirmed that the protection rate against HPV infection is
96\% 5 years after vaccination with HPV6, 11, 16 and 18-type quadruple vaccines and $100 \% 9.4$ years after vaccination with HPV16 and 18-type divalent vaccines. Given that cross-immunization between HPV types can decrease the infection rates of HPV31, HPV33, HPV45, HPV51 and HPV52, Tota et al. ${ }^{[19]}$ believed that the successful development of HPV vaccines has potentially decreased the likelihood of cervical cancer by $70 \%$.

Brabin et al. ${ }^{[20]}$ estimated that by $2025,70 \%-80$ of the $12-13$ year-old female population will be vaccinated against HPV, thus possibly decreasing the future incidence of cervical cancer in women aged $20-29$ years by $55 \%-$ $63 \%$. Koliopoulos et al ${ }^{[3]}$ also believed that if the vaccine is inoculated widely, the incidence of cervical cancer will be significantly reduced 20 years later.

\section{HPV-related cervical cancer screening}

HPV typing is a supplemental screening method that effectively improves the efficiency of cervical cancer screening. UK, US, South Africa, Thailand, and Germany advocate the inclusion of HPV testing in cervical cancer screening programs ${ }^{[21]}$. A nationwide audit report in Sweden showed that $65 \%$ of Swedish patients who have been diagnosed with cervical cancer have never participated in cervical cancer screening, and that only $25 \%$ of cervical cancer patients have participated in cervical cancer screening with HPV subtype test. Given that most cases of cervical cancer were diagnosed at an early stage in these patients, most had a good prognosis ${ }^{[22]}$.

Del Mistro et al. ${ }^{[23]}$ reported that compared with other screening methods, such as cytology screening and colposcopy screening, HPV screening for cervical cancer has the best performance and highest sensitivity (92.3\%) and specificity (78.6\%). Gyllensten et al ${ }^{[22]}$ found that the sensitivity difference between cytological screening and HPV subtype test among postmenopausal women is greater than that among women of other ages, whereas the sensitivity of HPV subtype screening is thrice that of cytological screening.

Saslow et al. ${ }^{[24]}$ reported a more detailed screening procedure for cervical cancer and proposed that cervical cancer screening should begin from the age of 21 . Women aged 21 to 29 should undergo cytological screening every 3 years. Women aged 30 to 65 should undergo cytology screening every 3 years or HPV test combined with cytological screening every 5 years. 
In conclusion, cervical cancer remains one of most serious malignant tumors that affect women's lives in the global context. In addition, high-risk HPV infection significantly promotes the development of cervical cancer. Understanding the distribution of HPV subtypes has guided the development of anti-HPV vaccines and the prevention of cervical cancer. At present, HPV vaccines are mainly inoculated in developed countries because of economic reasons. Future research on $\mathrm{HPV}$ and cervical cancer should aim to decrease the cost of HPV vaccines, improve the screening system for cervical cancer, and develop new approaches for the prevention of cervical cancer.

\section{Declarations}

\section{Acknowledgements}

No.

\section{Competing interests}

The author declares that he has no competing interest.

\section{Authors' contributions}

$\mathrm{T}$ Wang made the literature analysis and wrote, discussed and revised the manuscript of this review.

\section{References}

1 Denny L. Cervical cancer prevention: new opportunities for primary and secondary prevention in the 21st century. Int J Gynecol Obstet, 2012, 119(Suppl 1): 80-84.

2 Alam MS, Ali A, Mehdi SJ, et al. HPV typing and its relation with apoptosis in cervical carcinoma from Indian population. Tumour Biol, 2012, 33(1): 17-22.

3 Koliopoulos G, Valasoulis G, Zilakou E.An update review on HPV testing methods for cervical neoplasia. Expert Opinion on Medical Diagnostics, 2009, 3(2): 123-131.

4 Halfon $\mathrm{P}$, Benmoura D, Khiri $\mathrm{H}$, et al. Comparison of the clinical performance of carcinogenic HPV typing of the Linear Array and Papillocheck HPV-screening assay. J Clin Virol, 2010, 47(1): 38-42.

5 Ramogola-Masire D, McG rath CM, Barnhart KT, et al. Subtype Distribution of huma n papillomavirus in HIV-infected women with cervical intraepithelial neoplasia stages 2 and 3 in Botswana. Int J Gynecol Pathol, 2011, 30(6): 591-596.

6 Kjaer SK, Breugelmans G, Munk C, et al. Population-based prevalence, type-andage-specific distribution of HPV in women before introduction of an HPV-vaccination program in Denmark. Int J Cancer, 2008,123(8): 1864-1870.
7 Sanchez GI, Kleter B, Gheit T, et al. Clinical evaluation of polymerasechain reaction reverse hybridization assay for detection and identification of human papillomavirus type 16 variants. J Clin Virol, 2011,51(3): 165-169.

8 Abdelmutti N, Hoffman-Goetz L. Risk Messages about HPV, Cervical Cancer, and the HPV Vaccine Gardasil in North American News Magazines. J Cancer Educ, 2010, 25(3): 451-456.

9 Erica R, Heitmann, Diane M, et al. Prophylactic HPV Vaccines and Prevention of Cervical Intraepithelial Neoplasia. Current Obstetrics and Gynecology Reports, 2012, 1(3): 95-105.

10 Esmaeili M, Bonyadi M, Dastranj A, et al. HPV typing in women with cervical precancerous and cancerous lesions in northwestern Iran. Gynecol Obstet Invest, 2008, 66(1): 68-72.

11 Vinodhini K, Shanmughapriya S, Das BC, et al. Prevalence and risk factors of HPV infection among women from various provinces of the world. Arch Gynecol Obstet, 2012, 285(3): 771-777.

12 Gita Eslami, Maryam Golshani, Mohammad Rakhshan, et al. PCR detection and high risk typing of human papillomavirus DNA in cervical cancer in Iranian women. Cancer Therapy, 2008, 6A:361-366

13 McKenzie ND, Kobetz EN, Hnatyszyn J, et al. Women with HIV are more commonly infected with non-16 and -18 high-risk HPV types. Gynecologic Oncology, 2010, 116(3): 572-577.

14 Rahman M, Sasagawa T, Yamada R, et al. High prevalence of intermediate-risk human papillomavirus infection in uterine cervices of Kenyan women infected with human immunodeficiency virus. J Med Virol, 2011, 83(11): 1988-1996.

15 Cercato MC, Mariani L, Vocaturo A, et al. Predictors of human papilloma virus (HPV) infection in Italian women. J Med Virol, 2010, 82 (11): 1921-1927.

16 Nadarzynski T, Waller J, Robb KA, et al. Perceived risk of cervical cancer among pre-screening age women (18-24 years): the impact of information about cervical cancer risk factors and the causal role of HPV. Sex Transm Infect, 2012, 88(6): 400-406.

17 Wiley DJ, Masongsong EV, Lu S, et al. Behavioral and sociodemographic risk facto rs for serological and DNA evidence of HPV6, 11,16,18 infections[J]. Cancer Epidemiol, 2012, 36(3): 183-189.

18 Kepka,Berkowitz, Yabroff, et al. Human papillomavirus vaccine practices in the USA: do primary care providers use sexual history and cervical cancer screening results to make HPV vaccine recommendations. Sex Transm Infect, 2012, 88(6): 433-435.

19 Tota J, Mahmud SM, Ferenczy A, et al. Promising strategies for cervical cancer screening in the post-human papillomavirus vaccination era. Sex Health, 2010, 7(3): 376-382.

20 Brabin L, Kitchener HC, Stern PL. Implementation of prophylactic HPV vaccination:progress and future challenges. Expert Review of Obstetrics and Gynecology, 2010, 5(5): 591-603.

21 Chao A, Hsu KH, Lai CH, et al. Cervical cancer screening program 
integrating Pap smear and HPV DNA testing:a population-based study. Int J Cancer, 2008, 122(12): 2835-2841.

22 Gyllensten U, Sanner K, Gustavsson I, et al. Short-time repeat highrisk HPV testing by self-sampling for screening of cervical cancer. $\mathrm{Br} \mathrm{J}$ Cancer, 2011, 105(5): 694-697.

23 Del Mistro A, Frayle-Salamanca H, Trevisan R, et al. Triage of women with atypical squamous cells of undetermined significance (ASC-US): results of an Italian multicentric study. Gynecol Oncol, 2010, 117(1): 77-81.

24 Saslow D, Solomon D, Lawson HW, et al. American Cancer Society, American Society for Colposcopy and Cervical Pathology, and American Society for Clinical Pathology screening guidelines for the prevention and early detection of cervical cancer. J Low Genit Tract Dis, 2012, 16(3): $175-204$. 